Meta

Journal des traducteurs

Translators' Journal

\title{
Approche de la dénomination en langue spécialisée
}

\section{Myriam Bouveret}

Volume 43, numéro 3, septembre 1998

URI : https://id.erudit.org/iderudit/002130ar

DOI : https://doi.org/10.7202/002130ar

Aller au sommaire du numéro

Éditeur(s)

Les Presses de l'Université de Montréal

ISSN

0026-0452 (imprimé)

1492-1421 (numérique)

Découvrir la revue

Citer cet article

Bouveret, M. (1998). Approche de la dénomination en langue spécialisée. Meta, 43(3), 393-410. https://doi.org/10.7202/002130ar

\section{Résumé de l'article}

Cet article s'interroge sur l'adéquation de la théorie de la terminologie en relation avec les pratiques actuelles de la discipline et propose quatre hypothèses reposant sur des études conduites en biotechnologie et en bioinformatique. L'auteur démontre que les termes sont bien des signes linguistiques. Ils en sont cependant différents car leur sens est réglé par un contexte linguistique et pragmatique tout autant que par un système conceptuel. La tâche désormais dévolue à la terminologie est de comprendre la nature linguistique des contextes spécialisés afin d'être en mesure d'analyser le sens des termes. 


\title{
APPROCHE DE LA DÉNOMINATION EN LANGUE SPÉCIALISÉE ${ }^{*}, 1$
}

\author{
MYRIAM BOUVERET ${ }^{2}$ \\ Praxiling, Université Paul-Valéry, Montpellier, France
}

\begin{abstract}
Résumé
Cet article s'interroge sur l'adéquation de la théorie de la terminologie en relation avec les pratiques actuelles de la discipline et propose quatre hypothèses reposant sur des études conduites en biotechnologie et en bioinformatique. L'auteur démontre que les termes sont bien des signes linguistiques. Ils en sont cependant différents car leur sens est réglé par un contexte linguistique et pragmatique tout autant que par un système conceptuel. La tâche désormais dévolue à la terminologie est de comprendre la nature linguistique des contextes spécialisés afin d'être en mesure d'analyser le sens des termes.
\end{abstract}

\section{Abstract}

This paper calls into question the theory of terminology and proposes four hypotheses based on the study of specialized lexicons in biotechnology and biocomputing. It shows that the actual task of terminology is to understand the linguistic nature of the specialized context and the way the meaning is constructed. It concludes that terms are linguistic signs but are different from them not because they are monosemic by nature but because their meaning is constrained by their specialized linguistic and pragmatic context as much as by their conceptual domain.

\section{PRÉSENTATION}

En terminologie, le terme est distingué de la dénomination de langue générale, et les études appliquées à l'intelligence artificielle, à la traduction ou à la terminographie visent à poser une frontière entre les deux, tendant ainsi à renforcer ce fonctionnement idéal du terme. L'étude des termes est envisagée selon une organisation en systèmes conceptuels et leur fonctionnement dans ce cadre est généralement analysé comme indépendant des locuteurs, des discours et des conditions de production. Cette approche résulte d'une nécessité première de la terminologie : la normalisation. La terminologie classique viennoise, selon la tradition wüstérienne, s'est développée selon quatre grands principes qui sont le fruit d'un raisonnement déductif, contraint par cette nécessité. En effet, la terminologie a pour objet de créer des vocabulaires aidant à normaliser des pratiques de spécialistes. Son ennemi est donc l'ambiguïté. Pour remédier à cela, les dénominations sont construites et hiérarchisées selon un premier principe :

1) les dénominations en terminologie forment un système de classification à l'image des taxinomies. Or, si l'on veut se situer à l'intérieur d'un tel système, cela suppose d'envisager l'ensemble d'un domaine, de le délimiter comme un domaine clos où les dénominations peuvent être hiérarchisées et situées sans ambiguïté les unes par rapport aux autres. Cela implique d'isoler les domaines de spécialité les uns des autres et de les couper de la langue générale, car ce sont des classifications possédant une cohérence interne, dans un système clos. On est donc conduit par cette nécessité impérative à fonder la terminologie sur un second principe :

Meta, XLIII, 3, 1998 
2) il existe une langue générale et une sous-langue : la langue de spécialité, ellemême divisée en domaines fermés. Ces deux nécessités premières de la terminologie entraînent donc d'emblée à poser que le terme est distinct du signe de la langue naturelle. Et les deux principes suivants sont les conséquences théoriques logiques de ces nécessités. En effet, une fois établi ce fonctionnement du terme selon une classification intrinsèque, toute ambiguïté pouvant le toucher est logiquement éliminée, grâce d'une part à un fonctionnement selon lequel le terme est situé de manière différentielle par rapport aux autres, et d'autre part parce que chaque terme du domaine perd son lien avec les autres signifiants identiques, dans d'autres domaines ou en langue générale. Ainsi, la désambiguïsation du terme repose sur l'évacuation de l'éventuelle polysémie par un principe d'homonymie. La monosémie du terme est alors assurée parce qu'à chaque signifiant du domaine est associé un signifié unique. C'est là le troisième principe de la terminologie :

3) le terme est monosémique. À l'intérieur de ce système de classification où chaque entité, nommée concept, renvoie à une seule réalité et se situe différentiellement par rapport aux autres, le concept prend les propriétés d'un signifié. Tous deux correspondent à un ensemble de sèmes définis différentiellement par rapport aux autres entités du classement. C'est ce fonctionnement qui entraîne le quatrième principe :

4) le concept est l'équivalent du signifié.

Nous montrerons les difficultés d'une réflexion linguistique selon ces principes et proposerons quatre hypothèses contraires :

1) Les systèmes de classification ne font pas du terme un taxon, ni un nom propre, ni une étiquette. Les termes opèrent une catégorisation tout comme les autres dénominations.

2) Le terme n'est pas monosémique, mais seul un «réglage de sens forcé» (Siblot 1989) évite les dysfonctionnements de sens.

3) Il n'existe pas une langue générale et une langue de spécialité divisée en domaines, mais le terme est un signe dont le fonctionnement est spécialisé par le contexte de référence

4) Le concept n'est pas un simple signifié, mais une construction de connaissances.

À travers cet article, nous souhaitons montrer que le terme n'est pas différent du signe de langue commune. Tout comme celui-ci, il est une dénomination, mais dont la spécificité est d'être fortement normé, ce qui conduit injustement à le considérer comme un nom-étiquette, un nom propre ou à l'assimiler à un taxon.

\section{LE TERME N'EST NI UNE ÉTIQUETTE, NI UN TAXON, NI UN NOM PROPRE}

Le terme situé dans un système notionnel doit avoir une signification univoque. Les termes sont considérés comme des «mots justes» et leur sens est institué en «sens propre» selon une tradition rhétorique n'admettant ni l'écart ni le sens figuré ${ }^{3}$. Le sens des termes en terminologie est envisagé par rapport au système notionnel. Il s'agit d'un classement onomasiologique de concepts : une onomasiologie. Les termes dans ce cadre ne sont pas considérés selon leur contexte de phrase et encore moins selon le contexte de production; ils sont réduits à des noms-étiquettes. Cet idéal de signification univoque du terme correspond à ce que Rastier met en évidence comme une quête de l'orthonyme :

Philosophes et grammairiens font depuis le Cratyle le rêve de l'orthonymie, désignation correcte et directe. Les Stoïciens estimaient que le nom tombe droit de la pensée vers ce qu'il désigne, et comparaient cette chute à celle d'un stylet qui se fiche droit (orthon) dans le sol (cf. 
Lallot, 1989, p. 141). Dernier en date, Pottier définit ainsi l'orthonyme : «mot juste» (1992, p. 42), désignation privilégiée, immédiate. (1992, p. 123) [...] Ensuite, il faut s'assurer de l'univocité des noms, de manière à ce qu'ils désignent une chose, mais une seule. Aussi, un grand rêve d'univocité traverse notre tradition, que résume pour les Modernes le grand principe de Sanctius : «Unius vocis unica est significatio» (un mot, une et une seule signification). (Rastier 1996)

Les termes sont également souvent assimilés à des taxons. En taxinomie, la classification s'effectue selon une relation privilégiée d'hyponymie et d'hyperonymie. La métaphore sous-tendant l'organisation taxinomique est la généalogie avec des termes comme ceux de famille, individu, parent, etc. C'est sous la forme d'un arbre généalogique que sont représentées les taxinomies, ce qui renforce sans doute l'identification du terme au nom propre. Ces dénominations sont fortement réglementées. La normalisation des vocabulaires peut émaner des centres de recherches. Lors de colloques, un certain nombre d'interventions peuvent être consacrées à la mise au point de ces taxinomies. Les méthodes à l'heure actuelle dans l'établissement d'arbres taxinomiques en biologie sont liées à l'étude génomique. Elles ont recours également aux mathématiques et à l'informatique, afin de calculer l'évolution des espèces. Mais les méthodologies elles-mêmes donnent lieu à des controverses. En phylogénie, la méthode de l'échantillonnage est contestée et l'on considère qu'afin d'étendre le nom des individus identifiés à d'autres semblables, l'échantillon sur lequel ont porté les recherches doit être suffisamment élevé, évoquant «l'absence de fiabilité des phylogénies construites avec peu d'espèces» ${ }^{4}$.

Les taxinomies, en raison d'évolutions diverses, ne suivent pas forcément l'ordre parfait qu'on leur prête. Ainsi, le progrès des découvertes scientifiques ou les polémiques entre auteurs mettent en cause la validité de taxinomies déjà établies et conduisent à des remaniements fréquents. Il faut donc régulièrement procéder à des révisions et les vocabulaires sont alors constitués des anciens termes et des nouveaux, devenant synonymes.

Les taxinomies ne constituent qu'une partie des termes et l'on ne peut réduire le terme au taxon. Cependant, il existe un fonctionnement semblable entre ces deux systèmes classificatoires que sont la taxinomie et la terminologie : elles reconduisent toutes deux un vœu idéal d'adéquation entre le réel et la dénomination. Ainsi, le terme est souvent identifié et comparé au taxon, mais n'est pas un taxon. Sur la base d'un ensemble de corpus agro-alimentaires, nous avons pu établir une typologie des termes rencontrés. Ce corpus comprend un relevé de termes dérivés et composés à partir du terme lait d'après un suivi de 1990 à 1995 de la revue Le lait et de la Revue d'industrie agroalimentaire; le suivi d'une innovation

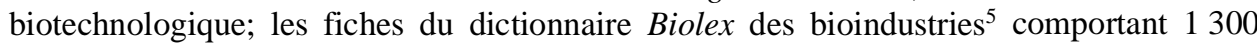
entrées. Ce corpus nous a permis de constater que l'ensemble des termes se répartit selon les catégories suivantes : les appareils, machines, dispositifs, ex. : les fermenteurs; les actions et réactions, ex. : l'acidification; les produits, ex. : les pâtes à tartiner; les qualités liées à ces produits, ex. : la tartinabilité. Une dernière catégorie est celle des nomenclatures et taxinomies ex. : les enzymes, les bactéries $^{6}$.

On peut dresser le tableau récapitulatif 1.

Concernant la formation des termes, certaines règles correspondent à ces catégories :

Les appareils ou machines sont la plupart du temps des syntagmes construits le plus souvent simplement par dérivation du terme de l'action correspondante ou bien parfois par transfert de la langue commune ou d'un autre secteur : ex.: fermentation > fermenteur, broyage > broyeur et ses dérivés : broyeur à couteaux, broyeur à congélation sous azote liquide, etc.

Les termes d'actions et de réactions peuvent être formés par exemple à partir des hyperonymes de nomenclatures, ex.: hydrolase > hydrolyse, où l'agent hydrolat est dénommé comme étant à l'origine d'une réaction; à partir d'une action technique : chauffage, 
Tableau1

Catégories de termes

\begin{tabular}{|l|}
\hline $\begin{array}{l}\text { appareils / machines / dispositifs } \\
\text { ex. : encapsuleuse }\end{array}$ \\
\hline actions / réactions \\
ex. : acidification \\
\hline produits \\
ex. : laitfermenté \\
\hline $\begin{array}{l}\text { qualités liées à ces produits } \\
\text { ex. : tartinabilité }\end{array}$ \\
\hline $\begin{array}{l}\text { nomenclatures / taxinomies } \\
\text { ex. : bactérie lactique }\end{array}$ \\
\hline
\end{tabular}

où l'action de chauffer est dénommée (en agro-alimentaire, il s'agit spécifiquement de chauffer le lait à $30^{\circ}$ ), la transparisation du lait qui rend le lait transparent, le traitement $d u$ lait à ultra haute température (UHT) utilisant la technique UHT.

Les nomenclatures, quant à elles, peuvent être des symboles, CO2; des produits, alcool; elles peuvent obéir à une formation gréco-latine : alcool déshydrogénase. Les taxinomies peuvent être des noms de plantes, d'animaux, de micro-organismes : bactérie, bactérie lactique elles peuvent obéir à une formation gréco-latine et s'il s'agit d'un taxon, le terme est écrit en italiques : Streptococcus lactis diacetylactis. Ce sont aussi souvent des éponymes. Les termes des taxinomies sont en italique et en latin et sont sujets à la siglaison, comme c'est le cas pour les symboles des nomenclatures. Le lactobacille, par exemple, est siglé $\mathrm{L}$ dans les termes composés et le terme Lactobacillus lacteus peut s'abréger en L. lacteus. Le sigle Lc, en revanche, est utilisé pour le terme simple lactobacillus.

Les produits sont le plus souvent des syntagmes composés de deux ou trois termes, ou davantage. Ils sont descriptifs de la composition : lait de brebis, lait de vache en poudre reconstitué, de la composition et du contenu : bouteille de lait stérilisé en plastique translucide, du destinataire : lait pour maman, des conditions : lait d'été, lait de citerne de ramassage.

Les qualités liées aux produits sont des adjectifs, lait transparent, aspect laiteux, des noms, des termes composés : opacité du lait. Les termes peuvent être des noms et s'insérer dans un paradigme dérivationnel : la tartinabilité, propriété d'être tartinable, ou la microondabilité, propriété d'être microondable.

Ces catégories ont été également répertoriées à partir d'un suivi de laboratoire auprès d'une équipe effectuant une recherche biotechnologique sur les arômes. L'industrie agro-alimentaire doit faire face à une perte des arômes naturels dans les aliments, dénaturés au cours des différents procédés. Afin de restituer cette perte d'arôme, ou de donner un arôme à un produit, on fait appel à des arômes fabriqués qui peuvent être de nature chimique. Les arômes obtenus par biotechnologies constituent un nouveau marché. Ils ont une grande valeur et un grand attrait parce que ce sont des arômes naturels. Le suivi des manipulations au cours d'une matinée de travail dans un laboratoire de biotechnologies ${ }^{7}$ a permis d'effectuer un relevé des termes tels qu'ils sont utilisés quotidiennement dans le cadre de la recherche : 
Des termes d'action: broyage, extraction, centrifugation, chromatographie pas de $g a z$, siglée en $C P G$ et abrégé à l'oral en «chromato pas de gaz», agitation et un hyponyme : agitation constante.

Des appareils, machines ou dispositifs : bain thermostaté, bécher, sonicateur, microscope, pipette stérile, réacteur, secoueur vortex, spectrophotomètre et l'action correspondante, terme dérivé : la spectrophotométrie, salle stérile, bec Bunsen, comptoir, boîte de Pétri, incubateur, étuve, flacon, paillasse, filtre, pissette, autoclaveur, balance.

La plupart de ces termes sont déjà connus car les expériences en biotechnologies des arômes ont déjà été conduites jusqu'à un certain stade dans ce type de travaux, mais une partie cependant est spécifiquement liée à cette recherche : enzyme, éthanol absolu, acide linoléique, acide gras, hydroperoxyde, alcool, composé aromatique volatil, gélose, microorganisme, huile, composé minéral, biomasse, eau distillée, tampon, bain de glace, protéine, enzyme, chloroforme.

On constate que les appareils ne sont pas des objets indifférents. Les termes d'appareils sont présents dans un travail de laboratoire, de même qu'ils sont représentés dans les publications où chaque étape de la recherche doit être décrite minutieusement afin de pouvoir être reproduite ou vérifiée dans les mêmes conditions.

Les nomenclatures et taxinomies ne constituent donc pas l'essentiel des termes comme l'ont montré nos corpus. Identifier le terme à un taxon est un artefact ne correspondant pas à la réalité des pratiques.

Une autre comparaison fréquente est celle consistant à apparenter le terme à un nom propre. Nous tenterons ici de déterminer si cette autre comparaison est valable. En terminologie scientifique et technique, on «donne un nom» à de nouvelles réalités, de nouveaux référents. Pour certains scientifiques, on «baptise» sa découverte. «Donner un nom» ou «baptiser», ces expressions suffisent-elles à identifier le terme au nom propre ? Sur la base de trois critères distinctifs entre le nom propre et le nom commun, on peut montrer que le terme n'est pas un nom propre.

Le premier critère est celui selon lequel il existe une relation de dénomination pour le signe alors qu'il s'agit d'une relation de désignation pour le nom propre. Ainsi, dans les exemples analysés par G. Kleiber (1984) :

Bernard est le nom du directeur d'École

Moucheron est le nom d'une petite mouche

Dans le premier cas, celui du nom propre, on peut substituer «est le nom de» par «renvoie», mais pas par «signifie». Dans le second cas, celui du nom commun, il s'agit d'une signification. «Moucheron désigne une petite mouche alors que Bernard renvoie au directeur d'école» (Kleiber 1984). Concernant le terme, si l'on considère la catégorie des termes substantifs, et ce même si l'on prend l'exemple d'un taxon qui tend le plus à se comporter comme un nom propre, on constate qu'il répond à la relation décrite pour le nom commun et pas à celle existant pour le nom propre. Le terme Escherichia Coli, par exemple, désigne une sorte de bactérie lactique, mais ne renvoie pas à une bactérie lactique.

Ainsi, même les taxons ne sont pas des noms propres et permettent une opération de catégorisation. La relation entre le référent et le nom est indirecte et passe par cette catégorisation. En l'occurrence, plusieurs individus peuvent prendre le nom de Escherichia Coli et ce nom leur est attribué sur la base de critères que le scientifique juge pertinents pour classer l'individu identifié comme étant une bactérie lactique de cette souche. En revanche, le nom propre que porte un individu n'est pas étendu à l'ensemble des individus possédant les mêmes propriétés typiques.

Et c'est un second critère de différenciation entre nom propre et nom commun : la particularisation du nom propre s'oppose à la catégorisation des noms communs. Or, le terme, 
qu'il s'agisse d'un taxon ou d'un autre type de terme, appartient toujours à une catégorie ou forme lui-même une catégorie. Cette «propriété individualisante» du nom propre n'est pas valable le concernant. Le nom propre en effet «réalise une identification individualisante» alors que «le nom commun réalise une identification catégorisante» (Siblot 1995b). On peut donc dire selon ce deuxième critère que le terme n'est pas un nom propre. Cependant, le désir de «labeliser» certaines découvertes, c'est-à-dire de rendre une réalité particulière par une nomination spécifique - ce fut le cas par exemple dans l'opposition de deux dénominations de laboratoires distincts pour le virus du sida, HIV ou HEPV3 - peut faire que le terme, dans certain cas, se rapproche du nom propre sans pour autant en emprunter le fonctionnement.

Le troisième critère que nous évoquerons ici est celui d'un sens direct ou indirect (Kleiber 1984 et 1996). La dénomination des noms propres est directe car le nom est attribué directement à l'individu en personne. La dénomination pour les noms communs est indirecte, elle se fait par l'intermédiaire d'une description. Ainsi, selon ce critère, la relation dénominative réalisée pour le terme est à l'image de celle du nom commun, indirecte, et repose sur une description. Cependant, on constate d'après ce critère, à quel point le désir de baptême, c'est-à-dire le fait de «labeliser» sa recherche par un nom, rapproche le fonctionnement du terme d'une relation dénominative directe telle qu'elle existe pour le nom propre. Et si l'on veut expliquer le rapprochement entre terme et nom propre, c'est sans doute sur la base de ce critère qu'il faut le faire, et voir dans ce parallèle le désir d'un pouvoir conféré par l'acte de nomination tel que l'a mis en valeur Siblot (1995a). Cet acte de nomination existe pour l'individu mais aussi pour les institutions. Le terme est donc pris entre cette double fonction: l'une effective de catégorisation et l'autre, idéale, de référence directe résultant d'une «parole nommante» (Siblot 1995a). Par ailleurs, si l'on considère comme Kleiber (1996) que la différence entre une relation directe et une relation indirecte réside dans le flou autorisé au locuteur («critères d'appartenance flous») concernant les noms communs, alors qu'il est totalement exclu pour le nom propre, on peut dire que le terme-substantif est bien un nom commun, mais que la volonté de contrôle sur les termes d'une part et que la catégorisation sous-jacente au modèle du terme selon des conditions nécessaires et suffisantes d'autre part ${ }^{8}$, tendent à exclure le flou en ce qui le concerne.

Sur la base de ces trois critères, on peut affirmer que le terme n'est pas un nom propre. Qu'il s'agisse de nomenclatures, d'actions, de réactions, d'instruments, de machines, les termes ne sont pas des désignateurs rigides contrairement aux noms propres. Et si la terminologie tend à cet idéal d'univocité entre la «chose» et le «nom», c'est pour mieux rejeter l'ambiguité inhérente à toute dénomination, spécialisée ou non, à des fins de normalisation. Le terme cependant est bien une dénomination, mais son fonctionnement est spécifique puisqu'il est d'une part normé par les spécialistes et que, d'autre part, ce sont les contextes qui transforment les mots en termes. C'est ce que nous verrons avec les deuxième et troisième hypothèses.

\section{LE TERME EST UN SIGNE}

La deuxième hypothèse met en question le principe de monosémie du terme en mettant en avant le fait que les dysfonctionnements que connaît le terme ne sont rien d'autre que le fonctionnement normal de tout signe de langue naturelle. Mais la diférence avec le signe est que le terme est normé au sein d'une communauté de spécialistes où il joue également un rôle de normalisateur institutionnel. De par sa fonction normalisatrice et par le consensus qui est institué au sein de la communauté où il est employé, le terme subit ce que l'on pourrait appeler un «réglage de sens forcé» (cf. Siblot 1989). Dans son emploi discursif chez les usagers spécialistes, plusieurs moyens sont mis en œuvre pour contrôler le sens des termes, 
y compris dans leur néologie. Ces procédés sont la création de termes par le jeu des racines gréco-latines, le recours à l'anglais comme code et, par ailleurs, la formation de termes par dérivation. De plus, le choix des termes est motivé par un certain dialogisme scientifique, une influence de la communauté à travers les circuits d'édition, les colloques, etc. Ce réglage de sens est l'ajustement du sens en actualisation, pour les besoins de la communication. Mais il s'effectue également en amont de l'actualisation, en néologie, puisqu'en effet le terme doit répondre à des critères de bonne formation. L'organisation des terminologies en systèmes conceptuels permet de régler le sens des termes, mais permet aussi de régler la formation et le sens de leur néologie. La théorie néologique de la terminologie correspond à la visée normalisatrice de la discipline et le principe majeur applicable à la formation des termes est le rejet des ambiguïtés. Plusieurs moyens sont alors mis en œuvre :

La première manière est de former des termes à l'image du système établi par la classification notionnelle, c'est-à-dire que la formation morpho-syntaxique doit contraindre le sens des termes à des signifiés prévus ou contrôlés. C'est ainsi que les termes doivent idéalement être en adéquation avec la définition : «Les principes applicables à la formation d'un terme complexe sont par conséquent avant tout ceux qui régissent la formation des définitions et des notions.» (Felber 1987). Et cela semble plus facilement atteint en utilisant des dénominations à formation gréco-latine. C'est donc un principe de compositionnalité qui est mis en œuvre dans le contrôle de la signification du terme puisqu'elle doit reproduire celle de la définition. Le terme gréco-latin a l'avantage d'être universel et d'être plus motivé que le terme de français courant : «Depuis les années 30, on s'est efforcé d'élaborer une clé de la terminologie internationale, qui consiste en morphèmes internationaux (racines et suffixes). Cette clé devrait faciliter la formation de termes internationaux.» (Felber 1987).

La seconde manière est de régler l'utilisation des termes. Dans les normes officielles ou tout document à caractère normatif, il est conseillé d'utiliser des termes monosémiques et mononymiques, c'est-à-dire qu'une seule notion est affectée à un terme et un seul terme à une seule notion. Cette règle de non-ambiguïté est appliquée également à l'emprunt, à une langue étrangère ou à un autre domaine. Considérer comme emprunt à la même langue un transfert de terme témoigne d'une partition entre domaines clos et cela est renforcé par la recommandation avancée :

Parfois, il se révèle utile d'attribuer un sens modifié à un terme dont l'usage est courant dans un autre domaine, pourvu que ce domaine soit suffisamment éloigné pour qu'on évite toute ambiguïté. Ce terme est appelé terme transféré. [...] Par exemple les termes «information» et «code» ont été introduits dans le domaine de la génétique avec des sens modifiés. (Felber 1987)

La troisième manière d'éviter les ambiguïtés est de maintenir fermement des frontières entre domaines. Concernant la polysémie, définie comme existante «lorsque des termes identiques sont affectés à des notions différentes qui sont étymologiquement ou sémantiquement liées» (Felber 1987), le moyen de la désambiguïser est de maintenir une frontière entre les domaines, ceux-ci devenant ainsi des systèmes notionnels distincts. Ainsi, la polysémie est résolue soit par la multiplication des domaines soit par celle des systèmes notionnels à l'intérieur d'un même domaine. C'est donc un principe d'homonymie qui désambiguïse la polysémie toutes les fois que cela est possible, homonymie qui affecte à des termes identiques «des notions différentes qui sont indépendantes l'une de l'autre» (Felber 1987). En biologie, par exemple, les termes de code, message selon cette perspective devraient être considérés comme des emprunts de la biologie au vocabulaire de la communication, et non comme des métaphores. La théorie terminologique, à travers l'interprétation des processus de néologie même, préfère réduire la métaphore à un signifiant nouveau plutôt que de l'envisager comme un signifié nouveau. Ceci constitue là encore un moyen de circonscrire la polysémie des signes en la désambiguïsant selon un principe d'homonymie. 
Mais en réalité, le terme est sujet à la synonymie et à la polysémie, à tous les «dysfonctionnements» que connaissent les dénominations de langue commune. Tout terminologue dans sa pratique en rencontre de nombreux cas. Le terme n'est donc pas intrinsèquement monosémique, et en cela distinct du signe.

\section{Cas de synonymie}

Les phénomènes de synonymie sont fréquents dans les articles de vulgarisation et dans le cadre de l'enseignement (cf. Jacobi 1989; Assal 1994). La synonymie met en parallèle plusieurs termes qui circulent en même temps. Cette synonymie peut être représentée par des termes existant à la fois en anglais et sous leur équivalent français, ou bien, comme c'est le cas dans l'exemple qui suit, deux termes français coexistent dont l'un est un emprunt partiel. Le terme batch, utilisé en biotechnologies, vient du syntagme anglais batch fermentation et a pour équivalents français : fermentation discontinue ou fermentation en batch.

Un autre cas de synonymie peut être la concurrence de dénominations entre laboratoires. Celle-ci n'a pas pour seule cause une concurrence symbolique liée à une valeur de label (comme ce fut le cas dans la compétition de laboratoires se traduisant par la concurrence entre les dénominations $H I V$ et $H E P V 3$ pour dénommer le virus du sida) mais peut aussi reposer sur une perception distincte comme l'illustre l'exemple qui suit.

Lors de travaux sur la mise au point d'un nouveau procédé de production d'arômes, la question que se sont posée J.-M. B. ${ }^{9}$ et son équipe est celle de la nature du milieu dans lequel ils travaillaient. Quel adjectif allaient-ils choisir pour le caractériser : solide, pâteux ou semi-solide? Trois dénominations s'avéraient possibles :

fermentation en milieu solide (a)

fermentation en milieu pâteux (b)

fermentation en milieu semi-solide (c)

Le syntagme (a) emploie l'adjectif solide, calque du terme anglais solid state fermentation. Solide est ici opposé à liquide pour exprimer qu'il n'y a pas d'eau libre dans ce processus, seulement des particules. Le sens de solide est donc celui de non liquide. Cependant, la représentation, que nous pourrions appeler prototypique, de la solidité qui est convoquée ici est celle de la dureté d'une matière solide. Or le milieu de fermentation dont il est question ici ne correspond pas au sens évoqué par solide puisqu'il a une consistance souple. La dénomination solide évoque une matière indéformable alors que la matière en question est déformable, d'où la contestation de cette dénomination de fermentation en milieu solide, jugée abusive.

Puisque le milieu est proche d'une certaine viscoélasticité, comme une pâte, le terme de milieu pâteux a été jugé préférable par quelques personnes du laboratoire. Ce terme est attesté en industrie agro-alimentaire. L'adjectif pâteux est utilisé pour les fromages, par exemple, en industrie laitière, où il est souvent question de la viscoélasticité de la pâte. Et c'est cette dénomination que J.-M. B. préférait. Mais, décidé à combattre l'appellation de milieu solide, qu'il considérait comme un abus de langage, il décida avec son équipe de l'appeler milieu semi-solide (c), désignant selon eux un état intermédiaire entre le solide et le liquide. C'est d'ailleurs cette dénomination qu'ils avaient utilisée lors de la rédaction d'un chapitre sur ce type de fermentations dans le cadre d'un ouvrage sur l'utilisation des moisissures édité aux Pays-Bas. Mais leur terme de fermentation en milieu semi-solide s'est vu transformé en solid state fermentation alors qu'il était parfaitement acceptable en anglais comme : semi-solid state fermentation. Finalement, probablement à la suite d'une discussion de J.-M. B. avec un collègue de l'extérieur qui, lui, avait adopté l'adjectif pâteux, l'équipe est revenue à l'appellation de fermentation en milieu pâteux. 
On voit ici que la différence de perception entre un milieu plus ou moins solide conduit à hésiter entre plusieurs termes. Pendant la période où le terme de fermentation semisolide était employé par l'équipe en concurrence avec le terme anglais de fermentation solide, on peut parler de synonymie. Celle-ci s'est instaurée par volonté de lutter contre un anglicisme. Mais si le terme a dû être abandonné, c'est en fait parce que semi-solide correspondait déjà à un autre usage, plus spécifique à l'enzymologie, où il caractérise un milieu à la fois liquide et solide comme des billes dans un liquide. En enzymologie, le terme de fermentation semi-solide était déjà en circulation, ainsi «semi-solide» renvoyait à une autre réalité. Mais la synonymie n'a pas été abandonnée pour autant, puisque c'est finalement le terme de fermentation en milieu pâteux qui a été choisi pour dénommer cette nouvelle technique très spécifique, mise au point dans le cadre de recherches dont l'enjeu est la fabrication d'arômes naturels par voie biotechnologique. On peut donc dire qu'à l'heure actuelle, deux termes synonymes circulent qui sont fermentation en milieu solide et fermentation en milieu pâteux. Tous deux correspondent à une même réalité mais témoignent d'un découpage du réel distinct : l'un pour lequel la consistance pâteuse peut être considérée comme solide et l'autre pour lequel il existe des consistances solides, pâteuses, semi-solides et liquides. Il faut ajouter à cela que ces recherches sont spécifiques à ce laboratoire et que derrière la dénomination se référant au milieu pâteux se cache toute la nouveauté du procédé de fabrication de l'arôme.

Ainsi, l'insistance à trouver le mot juste recouvre plusieurs motivations d'ordre cognitif (la perception), linguistique (la lutte contre l'anglais), mais aussi d'ordre d'un désir de reconnaissance scientifique (nouveau procédé de fabrication). Et c'est précisément sur la base de cette appellation de milieu pâteux que naîtra un néologisme sémantique désignant le nouvel appareil destiné à faire fermenter ce milieu : un pétrin, terme emprunté à la panification. C'est sur cette innovation technologique que reposera la création d'une entreprise de fabrication d'arômes naturels par voie biotechnologique.

La synonymie évoquée ici est une synonymie que l'on pourrait qualifier d'actualisation, c'est-à-dire qu'elle intervient en discours et non dans le système classificatoire des termes de la biologie ou de l'enzymologie. Cette synonymie, ou circulation parallèle de plusieurs termes, est révélatrice du fait que les termes ne sont pas que de simples fenêtres sur une classification, mais sont aussi des fenêtres sur des concepts en construction. L'enjeu de même que le flou dénominatif sont d'autant plus grands que ces concepts sont liés à une innovation. Ainsi, les termes spécialisés sont d'une part classés sous forme de lexiques et d'autre part actualisés en discours. Or, selon ce fonctionnement, le terme n'est pas différent du signe.

\section{Cas de polysémie}

Les termes observés dans leur fonctionnement en discours révèlent également l'existence d'une polysémie. Plusieurs cas se rencontrent. L'un, très fréquent, est l'utilisation d'un même terme dans deux spécialités distinctes. Lorsque les spécialités sont éloignées, il est habituel de parler d'homonymie, mais lorsque les spécialités sont proches, comme la médecine et la bactériologie, par exemple, il est difficile de ne pas évoquer la polysémie. Le terme parasite, par exemple, représente en médecine une classe, alors qu'en bactériologie il désigne une sous-classe. Comprendre de quel sens il s'agit requiert de connaître la sphère d'activités dans laquelle on se trouve et refuser toute autre signification possible, en particulier celle de la langue générale qui suit :

parasite :- Personne qui vit dans l'oisiveté, aux dépens de la société, alors qu'elle pourrait subvenir à ses besoins. - Perturbations dans la réception des signaux radioélectriques. (Dictionnaire Le Petit Robert, 1985) 
Le scientifique évolue dans son propre domaine de spécialité et toute autre signification de parasite est alors ignorée, considérant qu'il ne s'agit pas du même terme mais d'un autre appartenant à un domaine différent. Ici, pourtant, médecine et biologie sont très proches. Cependant, la polysémie est ignorée au profit de l'homonymie, or c'est uniquement le partage des connaissances d'un même contexte de spécialité qui permet cette identification. Un sens est actualisé dans un contexte de spécialité particulier, parmi l'ensemble des sens implicités autour des différentes expériences liées à parasite. On peut donc dire, d'une part, qu'interpréter le sens repose sur une connaissance partagée : «c'est ce savoir extra-linguistique qui seul permet la sélection et le réglage de sens» (Siblot 1989) et, d'autre part, que le contexte spécialisé joue à la fois le rôle d'isotopie discursive et le rôle du contexte de situation permettant de lever l'ambiguïté.

Un autre exemple de polysémie est le terme d'agitation en biotechnologies ${ }^{10}$. En français, il n'existe qu'un terme alors qu'en anglais, l'agitation correspond à stirring (a) ou à shaking (b). Deux fonctionnements de l'agitation sont révélés : dans le premier cas, traduit par (a), le réacteur est immobile et on entre alors le mobile d'agitation à l'intérieur. Si c'est une agitation mécanique ou par le gaz, on envoie de l'air. Dans le deuxième cas, traduit par (b), c'est le contenant qui est lui-même sur quelque chose qui bouge. En français, afin de lever cette ambiguité, on est contraint de construire une paraphrase explicative comme : agité sur table agitante, alors qu'en anglais un simple shaked suffirait à faire comprendre de quel dispositif d'agitation on parle. Les termes d'agitateurs subissent la même distinction : agitateur à champ magnétique : magnetic stirrer (a) et agitateur à mouvement de va-et-vient : to and fro shaker (b). L'agitateur (a) selon le catalogue OSI ${ }^{11}$, repose sur le principe suivant : «un système crée un champ magnétique tournant, qui engendre le mouvement du barreau aimanté, permettant d'homogénéiser la solution». C'est donc un cas d'agitation «interne» décrite par stirrer. L'agitateur (b) sert à aérer des cultures de microorganismes et le récipient subit un mouvement de va-et-vient. C'est ici un cas d'agitation «externe» exprimée par shaker.

Sans prétendre dresser une typologie exhaustive de la polysémie des termes spécialisés, nous voudrions proposer un classement de quelques grands types représentatifs des cas rencontrés dans nos corpus. La polysémie est multiple. Elle peut par exemple être liée à l'évolution de techniques, à des transferts de vocabulaires d'une discipline à l'autre, voire à des chevauchements sémantiques entre langue générale et discours spécialisés (cf. Candel 1984). Nous classerons ici six types de polysémie.

\begin{tabular}{|c|c|}
\hline Type 1 & $\begin{array}{l}\text { changement en diachronie par affinement des découvertes ou évolution des } \\
\text { techniques } \\
\text { ex. : virus }\end{array}$ \\
\hline Type 2 & $\begin{array}{l}\text { deux termes anglais, représentatifs de deux domaines et correspondant à deux } \\
\text { registres savant et courant, correspondent en français à un seul terme } \\
\text { ex. : fungus/mushroom } \\
\text { champignon }\end{array}$ \\
\hline Type 3 & $\begin{array}{c}\text { chevauchement entre langue générale et langue spécialisée } \\
\text { ex. : peuplement }\end{array}$ \\
\hline Type 4 & $\begin{array}{l}\text { deux niveaux de spécificité correspondent à des applications différentes } \\
\text { ex. : bactérie lactique }\end{array}$ \\
\hline Type 5 & $\begin{array}{l}\text { un hyperonyme et un hyponyme répondent à un même terme } \\
\text { ex. : parasite }\end{array}$ \\
\hline Туре 6 & métaphore \\
\hline & \\
\hline
\end{tabular}


Pour le type 1, le terme de virus, par exemple, en médecine a perdu désormais son sens premier très général (a) pour n'être plus utilisé en médecine ou biologie qu'au sens (b) $)^{12}$ :

- nom donné à tout germe pathogène (a)

- microorganisme infectieux parasite absolu des cellules vivantes possédant un seul type d'acide nucléique et se reproduisant à partir de son seul matériel génétique, à structure bien définie (b) (Dictionnaire Le Petit Robert, 1985).

Pour le type 2, dans l'exemple de champignon, c'est le contexte ou une paraphrase liée au terme qui permet de savoir de quelle notion il s'agit : de champignon de moisissure ou de champignon alimentaire. Les termes anglais fungus et mushroom font cette distinction en biotechnologies ${ }^{13}$.

Pour le type 3, le terme peuplement est ambigu dans une discipline comme la zoogéographie ${ }^{14}$. Son emploi peut en effet renvoyer au peuplement des espèces animales comme à la notion en démographie humaine de population selon l'acception en langue générale. Les définitions sont les suivantes :

- processus démographique par lequel un territoire reçoit sa population;

- état d'un territoire peuplé;

- ensemble des organismes animaux et végétaux vivant dans un même milieu biogéographique (Écol.) (Dictionnaire Le Petit Robert, 1985).

Le type 4 peut être illustré par le terme bactérie lactique recouvrant deux sens différents de «fermentaire» ou «homofermentaire» selon l'application aux produits laitiers ou au $v^{1}{ }^{15}$. Cet exemple sera repris et approfondi plus loin à propos du traitement de la polysémie dans le dictionnaire Biolex.

Pour le type 5, c'est le cas de l'exemple parasite évoqué plus haut: en médecine, il s'agit d'une classe alors qu'en bactériologie, c'est une sous-classe ${ }^{16}$.

On pourrait ainsi poursuivre la liste et ajouter un type 6 correspondant à la métaphore. Celle-ci pose en effet également le problème de la polysémie, parce qu'elle importe au moins un sème commun sur lequel s'opère le glissement. Il s'agit donc bien d'une polysémie. C'est le cas par exemple du terme virus utilisé en informatique comme métaphore du virus biologique. Or si l'on se réfère à la distinction classique entre polysémie et homonymie (Kleiber 1984; Martin 1972, 1979) c'est ce sème commun minimal qui permet de parler de polysémie alors qu'aucun sème n'est commun en homonymie.

Le terme spécialisé n'est donc à l'abri ni de la synonymie ni de la polysémie. Le fonctionnement du terme est identique à celui du signe : ni l'un ni l'autre ne permettent de réaliser une relation univoque entre la dénomination et le référent. On peut en conclure que le terme est bien un signe.

\section{CONTEXTES SPÉCIALISÉS ET CONSTRUCTION DU SENS}

\section{Les contextes spécialisés transforment le signe en terme}

Notre troisième hypothèse met en évidence le fonctionnement des termes à l'intérieur de contextes discursifs et pratiques qui lui confèrent sa spécialisation, davantage que des frontières délimitant des systèmes. Les termes sont des dénominations spécialisées et leur spécialisation est déterminée par des pratiques et des locuteurs eux-mêmes spécialisés. On prendra pour exemple le terme identité, utilisé en bioinformatique dans l'activité de comparaison de séquences d'ADN par interrogation de bases de données via Internet (cf. Bouveret et Gaudin 1996). Cette notion fait partie du vocabulaire de base en bioinfor- 
matique, et savoir dans quelle mesure la séquence qu'on a isolée en laboratoire est comparable à celles existant déjà et contenues dans les banques de données rapatriées est une opération courante. Les deux communautés, biologistes et informaticiens, utilisent donc le terme.

Du point de vue ${ }^{17}$ des biologistes, ce qu'il est important de comprendre, c'est le degré d'identité car cela renseigne sur le caractère génétique de la ressemblance. Ainsi, parler d'identité est simplement un indice mais ne suffit pas, on utilisera alors deux autres termes plus précis. Si la ressemblance est faible, c'est-à-dire portant sur moins de huit bases, on parlera de similarité et si la ressemblance est forte, portant sur plus de huit bases, on parlera d'homologie. Or lors d'une comparaison de séquences d'ADN, seule l'homologie est pertinente pour le biologiste. Elle porte au moins sur huit bases semblables et possède un caractère génétique puisqu'elle est révélatrice d'un ancêtre commun. Ainsi, la notion d'identité pour le biologiste est hypéronyme de deux hyponymes : homologie et similarité, mais seule l'homologie est biologiquement pertinente.

Du point de vue des informaticiens impliqués dans l'élaboration des logiciels pour réaliser la comparaison de séquences, la notion d'identité signifie simplement qu'il existe une ressemblance globale entre les deux séquences, et c'est ce que le logiciel de comparaison de séquences doit pouvoir permettre de repérer. Les termes d'identité, homologie et similarité ont alors un rôle de synonymes. Il ne s'agit pas d'être en mesure de comprendre la distinction, il est évident que les deux communautés, biologistes et informaticiens, sont capables de la faire, mais c'est simplement une question de pertinence (cf. Sperber et Wilson 1989), ou tout simplement de nécessité selon la situation : le biologiste a besoin de la distinction alors que l'informaticien n'en a pas besoin. Dans un cas, la distinction est pertinente alors que dans l'autre elle ne l'est pas.

Dans cet exemple, on peut constater que le terme d'identité est le même dans les deux spécialités, cependant que sa valeur (au sens saussurien) est différente. Selon les deux points de vue, il n'entre pas dans les mêmes oppositions.

Comme on vient de le voir avec l'exemple d'identité, afin d'interpréter le sens de ce terme, c'est le point de vue spécialisé qui intervient, c'est-à-dire la communauté concernée, biologistes ou informaticiens. Mais c'est aussi la situation, la tâche requise : quelle information recherche-t-on, une indication générale ou une réponse précise ? Le concept n'est plus alors envisagé seulement en langue comme une entité au sein d'un réseau sémantique, mais comme étant aussi déterminé par une pratique et des locuteurs spécialisés. Les connaissances mises en œuvre ne sont donc pas seulement celles de représentations de connaissances au sein d'un système conceptuel, mais sont aussi des connaissances extralinguistiques, des connaissances partagées par des communautés de locuteurs spécialisés. Cette interrogation sur le concept en relation avec les connaissances spécialisées est l'objet de notre quatrième hypothèse.

\section{Concept et construction de connaissances ${ }^{18}$}

Le concept tel qu'il est envisagé en terminologie, et en particulier dans les bases de connaissances, est identifié à un signifié, c'est-à-dire qu'il est une entité en langue. Chaque signifé a une valeur l'opposant aux autres signifiés. Avec l'exemple précédant du terme identité, nous avons constaté que cette approche du concept était en partie valable. Mais on a pu se rendre compte également que le sens était construit à travers d'autres relations que celles entretenues simplement à l'intérieur d'un système notionnel. Selon Kleiber (1981), c'est l'effacement du référent qui conduit à une adéquation entre signifié et concept. Ainsi, en terminologie, cette confusion résulte d'une position nominaliste où les termes sont considérés comme des nomenclatures. Mais si l'on replace les termes en discours, actualisés par des locuteurs, leur plus forte caractérisation relève d'un réglage 
selon une norme, dans une sphère de connaissances donnée. Ainsi, ce qu'on appelle terme, ce sont les dénominations simples ou complexes, lexicalisées, qui ont un présupposé d'existence pour la communauté concernée. Le terme est donc une unité lexicalisée ou en voie de le devenir (néologismes) au sein d'une activité donnée. De ce fait, toute dénomination d'un texte spécialisé, tout candidat terme, n'est pas nécessairement un terme. La dénomination de langue commune ne subit pas, elle, cette contrainte.

La construction du sens du terme serait donc à envisager à deux niveaux : en domaine (correspondant à la langue pour le signe) et en discours. On peut dire que la construction du sens du terme spécialisé se réalise dans une interaction entre langue et discours. Les connaissances liées au terme ne sont donc pas seulement celles du domaine, mais également des connaissances d'ordre pratique et discursif. Qu'est-ce que cette constatation entraîne concernant l'application terminologique ? Étant donné la grande diversité des besoins en terminologie, de la conception de bases de connaissances à la traduction, on peut se demander s'il est vraiment souhaitable de réduire le concept à un signifé ou au contraire de l'envisager comme une construction de connaissances. Il semblerait qu'une approche plus souple soit préférable, et particulièrement selon une perspective de traitement informatique des données terminologiques. Envisageons deux situations assez éloignées de besoins terminologiques : la création de bases de connaissances et la traduction. Dans le premier cas, on a affaire à une activité de désambiguïsation du sens, autrement dit, il s'agit de supprimer l'ambiguïté. Dans le second cas, en revanche, il s'agit de traduire le sens, c'est-à-dire le restituer avec toute sa complexité. Ainsi, dans un compromis entre théorisation et terminographie, ne pourrait-on pas envisager la nature variable d'un concept selon un continuum entre domaine et actualisation en discours ? Selon les besoins rencontrés, et selon le type de termes rencontrés, le concept s'identifie en effet davantage à un signifé ou à une construction dynamique en contexte discursif. Or ce fonctionnement entre langue et discours, s'il a pu être vérifié pour le terme est aussi celui valable pour le signe. La sémantique interprétative à cet égard parle de sèmes inhérents et de sèmes afférents (cf. Rastier 1987). Une nouvelle équivalence s'établit donc entre le terme et le signe : l'un comme l'autre construisent leur sens dans cette interaction entre langue et discours.

C'est à cette complexité et à cette variation dans la nature du concept que nous sommes confrontés lors de la rédaction de fiches de deux dictionnaires : l'un de bioinformatique (biologie, informatique et statistiques) ${ }^{19}$ et l'autre, Biolex, de biotechnologies. Nous y avons été confrontés à travers la résolution de la polysémie, ce qui n'est pas un hasard puisque la polysémie renseigne sur la structuration du sens à l'intérieur des catégories, autrement dit des concepts (cf. Rosch et Mervis 1975). Deux fiches permettront d'illustrer cette variation dans les concepts terminologiques : l'une extraite du dictionnaire de bioinformatique et l'autre de Biolex.

Le premier cas est celui du terme code. En bioinformatique, trois sortes de codes ont été rencontrés : le code informatique, le code alphabétique et le code génétique. Le premier s'identife au codage en bit, le second code correspond au codage en lettres ACTG des acides aminés et le troisième au code génétique de l'ADN. Ces trois sortes de codes constituent, selon la communauté spécialisée ou selon la tâche visée, trois points de vue sur le terme de code et peuvent chacun faire l'objet d'une fiche de dictionnaire, comme trois concepts distincts. Ainsi, trois fiches ont été réalisées : code 1, code 2 et code 3 . Ce traitement s'apparente à de l'homonymie et c'est le choix qui a été réalisé, bien que selon une réflexion strictement linguistique, les trois codes partagent un lien sémantique évident.

En revanche, un autre cas s'est avéré plus problématique. Il s'agit du terme bactérie lactique. Le champ d'étude de la bactérie lactique est encore très complexe. La maîtrise de l'activité de ce microorganisme est un enjeu pour les biotechnologies. De nouvelles souches de bactéries lactiques sont régulièrement identifiées et sur ces progrès reposent des 
innovations en agro-alimentaire appliquées aux yaourts, au vin, etc. On est donc déjà face à une première opposition vis-à-vis du modèle de catégorisation selon des conditions nécessaires et suffisantes : «les concepts ou catégories sont des entités aux frontières clairement délimitées» (Kleiber 1990). Concernant la bactérie lactique, plusieurs applications typiques sont apparues :

\{app. typ. 1\}fromage

\{app. typ. 2 \} précurseurs d'arômes

\{app. typ. 3$\}$ laits fermentés

\{app. typ. 4$\}$ vin et cidre

Mais distinguer plusieurs applications typiques ne conduit-il pas déjà à distinguer plusieurs entrées ? Le choix de l'inscription en une ou plusieurs fiches n'est pas simple. En fait, nous avons affaire avec la bactérie lactique à un concept large, un concept d'espèce au même titre qu'une fleur ou un fruit. Il s'agit d'une véritable catégorie. La définition donnée par Rosch de la catégorie s'applique ici :

Par catégorie on entend un certain nombre d'objets considérés comme équivalents, les catégories sont généralement désignées par des noms (ex. : chien, animal) (Rosch et Mervis 1976).

Si l'on s'en tient à une théorie en conditions nécessaires et suffisantes, la difficulté est de caractériser cette catégorie de façon typique selon les rubriques de la fiche, sachant qu'il existe autant d'actions et de résultats différents de la bactérie lactique qu'il y a de milieux différents. Le métabolisme de la bactérie lactique est variable. Or, choisir une seule entrée lexicale pour ce terme, c'est faire ce choix du niveau sémantique de la catégorie. Dans ce cas, les rubriques d'application typique, d'objet typique, d'action typique correspondent à ce niveau sémantique. Autrement dit, il faudrait, pour chacune des rubriques, retenir un ou plusieurs traits sémantiques typiques de la catégorie. Cela suppose que, quelle que soit la sorte de bactérie lactique (ex. bactérie lactique hétérofermentaire, lactobacille etc.), ou la souche de bactérie lactique (ex. : Leuconostoc, Lb. helveticus ou Lb delbrueckii subsp. bulgaricus etc.), l'action serait applicable. La catégorie de la bactérie lactique a un niveau d'abstraction assez élevé.

Ainsi, on pourrait retenir, selon une première solution, le critère décrit par Scriban : «la principale conséquence de la croissance des bactéries lactiques est la transformation des sucres en acide lactique» (Scriban 1988). Or cette propriété typique n'est pas valable en ce qui concerne le vin, pour lequel la conséquence est une désacidification par fermentation malolactique. La fermentation malolactique intervient aussi dans le cidre. Nous sommes ici devant un cas d'impossibilité vis-à-vis d'un modèle de catégorisation selon des attributs constituant le dénominateur commun de la catégorie. Et c'est la critique à l'égard du modèle en CNS :

L'appartenance d'une entité particulière à une catégorie répond au système du vrai ou faux : un x quelconque est un chien ou n'est pas un chien, selon qu'il satisfait ou non aux conditions critériales de la catégorie «chien» (Kleiber 1990a).

Si l'on veut considérer deux actions typiques qui sont en fait opposées, on peut être conduit, en seconde solution, à distinguer deux entrées pour les bactéries lactiques :

bactérie lactique (1)

\{app. typ.\}transformation des sucres en acide lactique

bactérie lactique (2)

\{app. typ.\}fermentation malolactique 
Cette solution pourrait être satisfaisante du point de vue traditionnel terminologique d'une partition en signifiés, car on serait conduit à distinguer deux concepts de bactérie lactique correspondant à deux signifiés distincts. Mais cette solution ne nous convient pas car cela introduirait une distinction qui n'est plus de l'ordre de la catégorie : distinction entre bactérie lactique et bactérie malolactique induisant que la bactérie malolactique n'est pas une bactérie lactique. Ne pas identifier le concept au strict signifié et choisir de ne pas réaliser deux fiches, c'est permettre ici à la catégorie bactérie lactique l'enregistrement d'éléments typiques qui ne sont pas nécessaires et suffisants puisqu'ils sont ici opposés.

D'une autre manière, comme on l'a esquissé plus haut, on pourrait considérer, en troisième solution, qu'il y a autant d'entrées lexicales possibles pour bactérie lactique qu'il y a d'applications typiques, d'objets typiques et d'actions typiques. Nous distinguerions alors la bactérie lactique appliquée au fromage, celle appliquée aux laits fermentés, celle appliquée aux précurseurs d'arômes. De la même manière, associés à ces applications typiques distinctes, nous serions en mesure d'adjoindre des actions typiques et des objets typiques pour chaque fiche liée à une application typique. On distinguerait donc autant de signifiés de bactérie lactique qu'il y a d'applications typiques, quel que soit le niveau sémantique : catégorie, c'est-à-dire espèce, ou bien individu, c'est-à-dire souche. Ce choix de présentation du terme bactérie lactique impliquerait qu'il existe autant de catégories sémantiques de bactérie lactique que l'on aurait distingué de signifiés.

La solution finalement choisie a été celle d'une fiche unique du terme bactérie lacti$q u e$, respectant par là son rang sémantique de catégorie. Autrement dit, on ne considère qu'un concept. À l'intérieur de cette fiche, nous distinguons autant d'activités typiques et d'applications typiques qu'il est nécessaire pour la caractériser.

\{act. typ. 1$\}$ acidification

\{app. typ. 1\}fromage

\{act. typ. 2\} protéolyse

\{app. typ. 2 \} précurseurs d'arômes

\{act. typ. 3\} production de polysaccharides

\{app. typ. 3\}laits fermentés

\{act. typ. 4\}fermentation malolactique

\{app. typ. 4$\}$ vin et cidre

Ces deux exemples de code et de bactérie lactique montrent que selon les besoins ou le niveau de description choisi la tâche requise, on peut faire le choix d'une fiche unique ou de plusieurs fiches, c'est-à-dire d'un ou de plusieurs concepts. Le traitement terminographique peut donc refléter le choix linguistique de l'homonymie ou de la polysémie. Concernant un traitement terminographique manuel, ce choix est réalisable, mais l'est-il d'un point de vue informatique ? La polysémie constitue un moyen d'accès à une compréhension de la structuration des concepts en terminologie. Une étude sur les sortes de polysémie et d'ambiguïté permettait sans doute d'aider à comprendre la diversité de la nature des concepts terminologiques et d'envisager ainsi un traitement informatique adapté à des besoins variés : prise en compte du contexte d'énoncé et, dans ce cas, intervention de la syntaxe et de la sémantique dans la construction de sens du terme, ou bien limitation au signifié et, dans ce cas, le marquage du lexique renvoyant à l'ontologie suffit. 


\section{CONCLUSION}

La conclusion que l'on peut tirer de ces quatre hypothèses est la suivante : l'affirmation de la terminologie selon laquelle le terme et le signe sont différents en soi est réductrice d'un point de vue linguistique. Le terme est bien un signe, mais il a un fonctionnement spécifique, normé. Or cela implique que le terme ne produit pas du sens strictement à partir de sa place dans un système conceptuel, mais que le contexte, linguistique et extralinguistique, détermine aussi son sens. Il s'agit alors de comprendre dans un premier temps quelle est la nature des connaissances, c'est-à-dire comment la spécialisation du terme est marquée. Nous avons pu constater qu'elle l'était par son ancrage dans une activité spécialisée. On peut donc dire que, outre son inscription dans un domaine, la spécificité du terme repose sur un partage de connaissances spécialisées, par des locuteurs spécialisés. Mais cela ne suffit pas. Il faut aller plus loin, et ce sont là les pistes que nous envisageons à partir de cette conclusion. Il faut s'interroger sur la manière dont ces connaissances sont mobilisées en discours. Autrement dit, cela revient à comprendre le processus d'interaction entre langue et discours dans la construction du sens. Les questions suivantes sont alors impliquées. Comment la spécificité du terme est-elle marquée en contexte de discours ? Dans quelle mesure et comment le référent et les pratiques liées au terme sont-ils repérables dans un énoncé ?

Ces questions ne peuvent être résolues sans avoir recours à une analyse du contexte. La question centrale que l'on se posera alors est donc la suivante : qu'est-ce qu'un contexte spécialisé ? Comment celui-ci détermine-t-il les connaissances qui sont activées lors de l'interpétation d'un terme? Il ne s'agit pas seulement de comprendre comment se fait l'accès au lexique spécialisé, à l'ontologie, puisqu'il est établi que les connaissances ne sont pas simplement celles liées au domaine. Mais il s'agit de comprendre la dynamique du sens. Quelle est la nature et quel est le rôle du contexte spécialisé (contexte de discours et contexte extralinguistique) lors de l'interprétation des termes en énoncé ?

Pourquoi est-il nécessaire de comprendre cela ? Tout simplement parce que les termes n'apparaissent jamais isolés, sauf dans les listes de vocabulaires et qu'ils sont toujours à interpréter en énoncé, entourés d'autres unités linguistiques, au sein d'une phrase, d'un texte, et dans une situation particulière. Or cette problématique de l'interprétation des termes en contexte est valable dans tous les secteurs de la terminologie, tant pour la traduction automatique que pour la réalisation de dictionnaires ou de systèmes experts.

\section{Notes}

* L'auteur remercie chaleureusement François Gaudin pour sa lecture du présent article.

1. Cet article, sous une forme plus succincte, a fait l'objet d'une présentation au colloque TIA 97 organisé à Toulouse les 3 et 4 avril 1997.

2. Présentement stagiaire post-doctorale AUPELF-UREF, au laboratoire GRESLET, Université de Montréal, 1997-1998.

3. Pour une étude historique du sens propre et du sens figuré dans les tropes, voir Rastier (1994).

4. Hervé Philippe, «Phylogénie : le point de vue du biologiste», Actes de l'École thématique CNRS : Utilisations et développements de ressources informatiques en biologie moléculaire, 1995 : 33.

5. Dictionnaire réalisé au sein de l'UPRESA CNRS «Dynamiques sociolangagières», Université de Rouen dans le cadre d'un contrat avec la société Eurolux Computers, le ministère de la Recherche et la Délégation générale à la langue française.

6. Nomenclature est le terme utilisé en chimie et biochimie pour la classification systémique alors que taxinomie ou taxonomie (la voyelle de liaison peut être indiféremment un o ou un i) est le terme utilisé en biologie pour la classification des organismes vivants animaux, végétaux ou humains. Les nomenclatures utilisent un système de chiffres, de classes et sousclasses, etc., alors que les taxinomies utilisent un système arborescent sur un modèle généalogique en espèces, familles, etc.

7. Anonyme pour des raisons de confidentialité.

8. Pour une approche critique de la catégorisation lexicale en terminologie et une application aux représentations sémantiques dans les systèmes de terminologie, voir Otman (1995). 
9. Entretien avec J.M. B., directeur du laboratoire de biotechnologies de l'E. Pour des raisons de confidentialité, ni les noms propres ni les corpus d'enquêtes ne sont livrés.

10. Exemple extrait de Biolex, Dictionnaire des bioindustries réalisé au sein de l'UPRES-A CNRS «Dynamiques sociolangagières» Université de Rouen, fiche agitation.

11. Exemple extrait de Biolex, fiche agitateur.

12. Exemple extrait d'un entretien avec J. Oberti, directeur du laboratoire de virologie, Université des Sciences et Techniques du Languedoc, Montpellier II, février 1991.

13. Exemple extrait de Biolex, fiche champignon.

14. Exemple extrait d'un entretien avec J. Blanc, directeur du laboratoire de zoogéographie, Université PaulValéry, Montpellier III, février 1991

15. Exemple extrait de Biolex, fiche bactérie lactique.

16. Exemple extrait d'un entretien avec J. Oberti (id.).

17. La notion de point de vue en terminologie a été développée par A. Condamines et J. Rebeyrolle (1997).

18. Cette hypothèse d'un concept construit a été introduite par F. Gaudin dans un article intitulé «Terminologie, l'ombre du concept» (Gaudin 1996).

19. Travaux réalisés au sein de l'UPRESA CNRS «Dynamiques sociolangagières», Université de Rouen, dans le cadre d'un contrat avec la Délégation générale à la langue française.

\section{RÉFÉRENCES}

ASSAL, A. (1993) : «Synonymie et vocabulaires spécialisés», Le langage et l'homme, vol. 28, n 2-3, Bruxelles, DeBoeck Université, pp. 147-156.

BOUVERET, M. (1997) : «Le terme : une dénomination au sens réglé», Actes du colloque TIA 97, pp. 115-127.

BOUVERET, M. et F. GAUDIN (1996) : «Du flou dans les catégorisations, le cas de la bioinformatique», Actes du colloque Troisièmes Journées internationales de terminologie. Terminologie et interdisciplinarité, Bruxelles, 19-20 avril 1996, à paraître.

CANDEL, D. (1984) : «La polysémie en langue de spécialité», La Banque des mots, Paris, CILF.

CONDAMINES, A. et J. REBEYROLLE (1997) : «Point de vue en langue spécialisée», Meta, 42 (1), Presses de l'Université de Montréal, pp. 174-184.

FELBER, H. (1987) : Manuel de terminologie, Unesco, Infoterm.

FRANÇOIS, J. et G. DENHIÈRE (dir.) (1997) : Sémantique linguistique et psychologie cognitive. Aspects théoriques et expérimentaux, Presses Universitaires de Grenoble.

GAUDIN, F. (1993) : Pour une socioterminologie, des problèmes sémantiques aux pratiques institutionnelles, Publications de l'Université de Rouen, $254 \mathrm{p}$.

GAUDIN, F. (1996) : «Terminologie : l'ombre du concept», Meta, 41 (4), Presses de l'Université de Montréal, pp. 604-621.

JACOBI, D. (1989) : «Reformulation et transposition dans les manuels scientifiques», Cahiers du CRELEF, Université de Besançon, 28 (1-2), pp. 7-21.

KLEIBER, G. (1981) : Problèmes de référence : descriptions définies et noms propres, Klincksieck.

KLEIBER, G. (1984) : «Dénominations et relations dénominatives», Langages, Larousse, n 76, pp. 77-94.

KLEIBER, G. (1990) : La sémantique du prototype. Catégorie et sens lexical, PUF, coll. «Linguistique nouvelle».

KLEIBER, G. (1996) : «Noms propres et noms communs : un problème de dénomination», Meta, 41 (4), Presses de l'Université de Montréal, pp. 567-589.

LERAT, P. (1989) : «Les fondements théoriques de la terminologie», La Banque des mots, $\mathrm{n}^{\circ}$ spécial, CILF, pp. 51-62.

LERAT, P. (1995) : Les langues spécialisées, PUF, coll. «Linguistique nouvelle», 198 p.

MARTIN, A. (1972) : «Esquisse d'une analyse formelle de la polysémie», TraLiLi, vol. 1, n 10, Université de Metz-Strasbourg, pp. 125-136.

MARTIN, A. (1979) : «La polysémie verbale, esquisse d'une typologie formelle», TraLiLi, vol. 1, n 17, pp. 251261.

OTMAN, G. (1995): Les représentations sémantiques en terminologie. La modélisation des unités terminologiques sous la forme de réseaux sémantico-terminologiques, Thèse de doctorat en sciences du langage, dir. F. Rastier, Université Paris IV-Sorbonne, 357 p

RASTIER, F. (1994) : Sémantique interprétative, Paris, PUF.

RASTIER, F. (1994) : «Tropes et sémantique linguistique», Langue française, $\mathrm{n}^{\circ}$ 101, pp. 56-78.

RASTIER, F. (1996) : «Le terme entre ontologie et linguistique», à paraître.

ROSCH, E. et C. B. MERVIS (1975) : «Family Ressemblances: Studies in the Internal Structure of Categories», Cognitive Psychology, $\mathrm{n}^{\circ}$ 7, pp. 573-605.

SCRIBAN, R. (coord.) (1988) : Biotechnologie, Technique et documentation-Lavoisier. 
SIBLOT, P. (1989) : «Isotopie et réglage du sens», Cahiers de praxématique, $\mathrm{n}^{\circ} 12$, éd. Université Paul-Valéry Montpellier III, coll. «Langue et praxis», pp. 91-110.

SIBLOT, P. (1995a) : «Comme son nom l'indique...» Nomination et production de sens, Thèse de doctorat d'État en sciences du langage, Université de Montpellier III.

SIBLOT, P. (1995b) : «Noms et images de marque : de la construction du sens dans les noms propres», Nom propre et nomination, M. Noilly (dir.), Klincksieck, pp. 147-160.

SLODZIAN, M. (1993) : «La V.G.T.T. (Vienna general theory of terminology) et la conception scientifique du monde», Le langage et l'homme, De Boeck Université, vol. 28, n 4, pp. 223-233.

SLODZIAN, M. (1994) : «La doctrine terminologique, nouvelle théorie du signe au carrefour de l'universalisme et du logicisme», Alfa, 7-8, pp. 121-136.

SPERBER, D. et D. WILSON (1989) : La pertinence. Communication et cognition, Paris, Minuit (traduction de Relevance. Communication and Cognition, Oxford, Blackwell, 1986).

VERSTIGGEL, J.-C. (1997) : «Le rôle du contexte dans la construction de la signification», François et Denhière (dir.), Sémantique linguistique et psychologie cognitive. Aspects théoriques et expérimentaux, Presses Universitaires de Grenoble, pp. 19-50.

VICTORRI, B. et C. FUCHS (1996) : La polysémie. Construction dynamique du sens, Hermès.

WÜSTER, E. (1982) : «Exposé illustré et terminologique de la nomination du monde», Travaux de terminologie, n², Québec, GIRSTERM, Université Laval, pp. 27-76. 